\title{
Essential medicine selection during the COVID-19 pandemic: Enabling access in uncharted territory
}

T D Leong, ${ }^{1}$ BPharm, MSc (Med), MSc (Clin Epi); S M McGee, ${ }^{2}$ BPharm, BCom (Econ), PG Dip Health Econ, M Health Econ; A L Gray, ${ }^{3,4}$ BPharm, MSc (Pharm); R de Waal, ${ }^{3,5}$ MB ChB, MPH; T Kredo, ${ }^{3,6,7}$ MB ChB, MMed (Clin Pharm), PhD;

K Cohen, ${ }^{3,8}$ MB ChB, MMed (Clin Pharm), MSc (Epi), MCFP (SA), Dip HIV Man (SA), Dip Obst (SA);

G Reubenson, ${ }^{3,9}$ MB BCh, FC Paed (SA), DCH, DTM\&H; M Blockman, ${ }^{3,8}$ BPharm, MB ChB, PG Dip Int Res Ethics, MMed (Clin Pharm); J Nel, ${ }^{3,10}$ MB ChB, FCP (SA), Cert ID (SA), DTM\&H; G Maartens, ${ }^{3,8}$ MB ChB, MMed (Med), FCP (SA), DTM\&H;

H Rees, ${ }^{3,11}$ GCOB, OBE, MB BCh, MA (Cantab), MRCGP, DRCOG, DCH; R Wiseman, ${ }^{12}$ BPharm, MSc, PG Dip Health Economics; K Jamaloodien, ${ }^{1}$ BPharm, BCom (Law), MSc (Clin Epi); A G Parrish, ${ }^{3,13}$ MB ChB, DA (SA), FCP (SA), MMed (Med), MMedSci (Clin Epi)

${ }^{1}$ Essential Drugs Programme, Affordable Medicines Directorate, National Department of Health, Pretoria, South Africa

${ }^{2}$ South African Medical Association, Pretoria, South Africa

${ }^{3}$ National Essential Medicines List COVID-19 subcommittee, National Department of Health, Pretoria, South Africa

${ }^{4}$ Division of Pharmacology, Discipline of Pharmaceutical Sciences, School of Health Sciences, University of KwaZulu-Natal, Durban, South Africa

${ }^{5}$ Centre for Infectious Disease Epidemiology and Research, School of Public Health and Family Medicine, Faculty of Health Sciences, University of Cape Town, South Africa

${ }^{6}$ Cochrane South Africa, South African Medical Research Council, Cape Town, South Africa

${ }^{7}$ Division of Clinical Pharmacology, Department of Medicine, Faculty of Medicine and Health Sciences, Stellenbosch University, Cape Town, South Africa

${ }^{8}$ Division of Clinical Pharmacology, Department of Medicine, Faculty of Health Sciences, University of Cape Town, South Africa

${ }^{9}$ Department of Paediatrics and Child Health, Faculty of Health Sciences, University of the Witwatersrand, Johannesburg, South Africa; and Rahima Moosa Mother and Child Hospital, Johannesburg, South Africa

${ }^{10}$ Department of Internal Medicine, School of Clinical Medicine, Faculty of Health Sciences, University of the Witwatersrand, Johannesburg, South Africa

${ }^{11}$ Wits Reproductive Health and HIV Institute, Faculty of Health Sciences, University of the Witwatersrand, Johannesburg, South Africa

${ }^{12}$ Liberty Health (Pty) Ltd, Cape Town, South Africa

${ }^{13}$ Department of Internal Medicine, Faculty of Health Sciences, Walter Sisulu University, Mthatha, South Africa; and Frere and Cecilia Makiwane hospitals, East London, South Africa

Corresponding author: A L Gray (graya1@ukzn.ac.za)

The COVID-19 pandemic requires urgent decisions regarding treatment policy in the face of rapidly evolving evidence. In response, the South African Essential Medicines List Committee established a subcommittee to systematically review and appraise emerging evidence, within very short timelines, in order to inform the National Department of Health COVID-19 treatment guidelines. To date, the subcommittee has reviewed 14 potential treatments, and made recommendations based on local context, feasibility, resource requirements and equity. Here we describe the rapid review and evidence-to-decision process, using remdesivir and dexamethasone as examples. Our experience is that conducting rapid reviews is a practical and efficient way to address medicine policy questions under pandemic conditions.

S Afr Med J 2020;110(11):1077-1080. https://doi.org/10.7196/SAMJ.2020.v110i11.15271

The COVID-19 pandemic has posed unprecedented challenges for healthcare globally. Clinicians and policymakers have had to make urgent decisions regarding therapeutic interventions in the face of rapidly evolving evidence of variable quality. Some publications have become available as preprints prior to peer review, ${ }^{[1]}$ while others have been retracted following concerns raised regarding data reliability. ${ }^{[2,3]}$ To date, much of the preliminary evidence for new or repurposed interventions is from observational studies that are subject to bias and confounding, or from randomised controlled trials (RCTs) with limitations. RCTs of potential COVID-19 treatments are often unblinded and under-powered, and may report endpoints of limited clinical or local relevance. ${ }^{[4]}$ Preliminary RCT results, even from apparently high-quality trials, may be reversed by the accrual of subsequent information. ${ }^{[5]}$
The South African (SA) National Essential Medicines List Committee (NEMLC) is a ministerially appointed, non-statutory advisory committee responsible for development and management of the national Essential Medicines List (EML) and Standard Treatment Guidelines (STGs). ${ }^{[6]}$ Medicine selection for the STGs is based on principles of equity, evidence-based medicine, public health relevance, safety, effectiveness, cost-effectiveness, affordability and implications for practice. The STGs and EML are reviewed on an iterative basis, using an extensive peer review process. NEMLC decisions inform provision of medication in the public sector. Public sector standard of care frequently informs Prescribed Minimum Benefits entitlements in the private sector. ${ }^{[7,8]}$

In March 2020, an NEMLC COVID-19 subcommittee was formed to address the need for rapid appraisal and synthesis of evidence 
in order to inform COVID-19 treatment guidelines. The subcommittee conducts accelerated evidence reviews and provides recommendations to the national COVID19 Clinical Guideline Writing Committee, which in turn produces national guidelines on the clinical management of suspected or confirmed COVID-19 disease, issued by the National Department of Health (NDoH)/ National Institute of Communicable Diseases. ${ }^{[9]}$

We describe the rapid review process developed by the COVID-19 subcommittee using corticosteroids and remdesivir reviews to illustrate the evidence-to-decision (EtD) framework used to arrive at a recommendation.

\section{Rapid reviews}

Systematic reviews of high-quality RCTs are considered the pinnacle of evidence and are increasingly used to inform clinical guidelines, and health and social care policies. ${ }^{[10,11]}$ Systematic reviews use transparent and explicit methods to identify, select, critically appraise and synthesise data from relevant primary research based on $a$ priori protocols. Full systematic reviews can take months, or even years, to complete.

In the context of a pandemic, there is an urgent need for rapidly synthesised and appraised evidence to inform policy decisions. ${ }^{[12]}$ Rapid systematic reviews are a simplified but rigorous process to synthesise relevant evidence within a short period of time.

The rapid review process is outlined in Fig. 1. We developed a standard guidance document based on evolving methods from the Cochrane Rapid Reviews Methods Group. ${ }^{[13,14]}$ When a topic is identified, the subcommittee defines the question and scope of the review by specifying the population (e.g. hospitalised or ambulatory), intervention and comparison characteristics, types of studies that are eligible for inclusion, and importantly, the outcomes that are relevant to inform a policy decision (the PICO - population, intervention, comparison and outcomes framework is used). A lead reviewer from the subcommittee oversees the process, and independent reviewers with experience in conducting evidence syntheses may be co-opted to assist (e.g. members of technical expert review committees of NEMLC ${ }^{[6]}$ and the South African GRADE Network $\left.{ }^{[15]}\right)$. All reviewers complete standardised conflict of interest and confidentiality forms. The aim is to complete an initial draft version of a rapid review report within a week

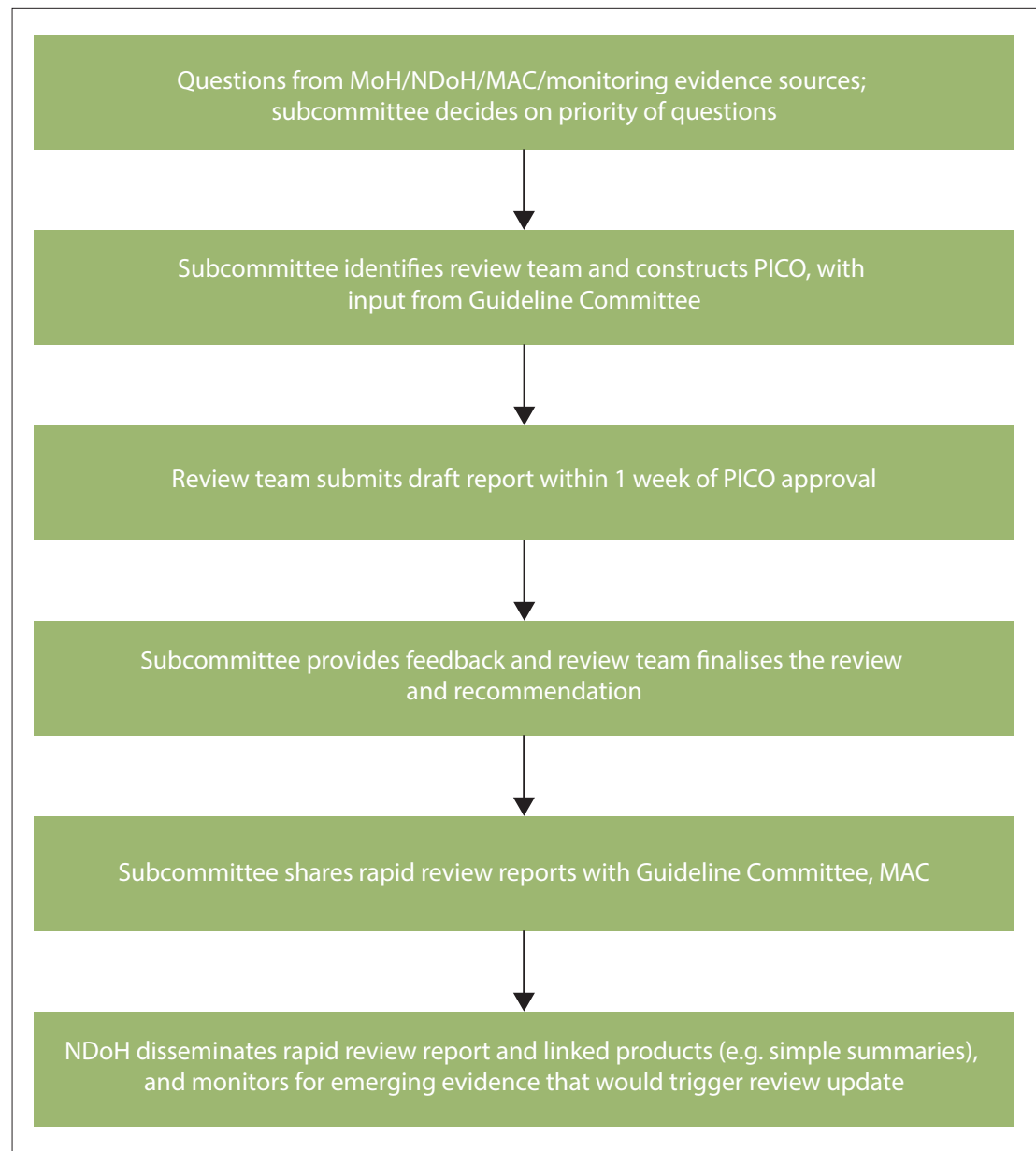

Fig. 1. Steps in conducting a rapid review for the COVID-19 guideline processes. $(\mathrm{MoH}=$ Minister of Health; $\mathrm{NDoH}=$ National Department of Health; $M A C$ = ministerially appointed committee; PICO $=$ the population, intervention, control and outcomes to be considered in the evidence review.)

(Fig. 1). If additional clinically relevant data on a previously reviewed product become available, rapid re-evaluation is undertaken.

The subcommittee reviews the evidence and uses an explicit EtD framework to make recommendations. ${ }^{[16,17]}$ EtD frameworks were developed as part of the Grading of Recommendations Assessment, Development and Evaluation (GRADE) approach to support systematic and transparent consideration of all factors relevant for a clinical or public health recommendation. ${ }^{[18]}$ The EtD framework has three main components: a clearly formulated question; assessment of the evidence and additional considerations for each decision criterion; and a final recommendation. ${ }^{[19]}$

The criteria applied in the EtD framework are quality of evidence, balance between benefit and harm, feasibility, infrastructure/ resource use, variability in stakeholders' values and preferences, and healthcare equity. These factors influence the strength of the recommendation, as shown in Table $1 .^{[19]}$
Importantly, all rapid reviews and recommendations from the NEMLC COVID19 subcommittee are placed in the public domain and can be accessed on the $\mathrm{NDoH}$ website. ${ }^{[20]}$ Acceptance and implementation of the guidelines depends on the level of trust and confidence clinicians have in the reliability and transparency of the process followed. To date, the subcommittee has reviewed 14 potential COVID-19 treatments (of which 3 were subsequently updated), and 4 are currently underway.

\section{Remdesivir for the treatment of severe COVID-19}

Remdesivir is an antiviral that inhibits viral RNA polymerases and has broad-spectrum activity against several virus families, including filoviruses (e.g. Ebola) and coronaviruses (e.g. severe acute respiratory syndrome coronavirus (SARS-CoV) and Middle East respiratory syndrome coronavirus (MERS$\mathrm{CoV}))^{[21-23]}$ It is being investigated as a potential treatment for COVID-19. 
Table 1. Criteria that inform recommendations ${ }^{[19]}$

\begin{tabular}{ll}
\hline Criteria & Description and link with strength of a recommendation \\
\hline Problem & The problem is determined by the importance and frequency of the healthcare issue that is addressed \\
(burden of disease, prevalence, or baseline risk). If the problem is of great importance, a strong & recommendation is more likely to be made. \\
& This describes how important health outcomes are to those affected, their variability and any related \\
uncertainty. & The higher the certainty in the evidence, the more likely it is that a strong recommendation will be made. \\
Values and preferences & The greater the net benefit or net harm, the more likely it is that a strong recommendation for or against the \\
Certainty of the evidence & option will be made. \\
Health benefits and harms and & This describes how resource-intense an option is, if it is cost-effective and if there is incremental benefit. The \\
burden and their balance & more advantageous or clearly disadvantageous these resource implications are, the more likely it is that a \\
Resource implications & strong recommendation will be made. \\
& The greater the likelihood of an option reducing inequities or increasing equity and the more accessible it is, \\
the more likely it is that a strong recommendation will be made. & The greater the acceptability of an option to all or most stakeholders, the more likely it is that a strong \\
Equity & recommendation will be made. \\
Acceptability & $\begin{array}{l}\text { The greater the feasibility of implementation of an option (specifically in the local context) to all or most } \\
\text { stakeholders, the more likely it is that a strong recommendation will be made. Where there are key barriers } \\
\text { to implementation of an option, these should be addressed. }\end{array}$
\end{tabular}

The review team searched two electronic databases (PubMed and Epistemonikos) and the Cochrane living systematic reviews website, and checked the clinicaltrials.gov database for ongoing studies.

The review identified two RCTs that compared remdesivir with usual care. Both were terminated early: one for challenges with recruitment and the other as further randomisation was considered unnecessary. ${ }^{[24,25]}$ Both trials found that remdesivir had no impact on mortality, with no increase in adverse events. One trial showed that remdesivir was associated with a reduction in median time to recovery, from 15 to 11 days. ${ }^{[25]}$

In the absence of a mortality benefit, the subcommittee considered reduction in intensive care unit (ICU) stay to be the most relevant clinical outcome, given SA's limited ICU bed capacity. However, there are as yet no data showing that remdesivir reduces ICU admission duration, or prevents progression to invasive ventilation. Preliminary results of the ACTT-1 (Adaptive Covid-19 Treatment Trial) study showed modest benefit among patients hospitalised for hypoxia, with a difference in median time to recovery of 2 days ( 7 v. 9 days; rate ratio $1.47 ; 95 \%$ confidence interval $1.17-1.84$ ), but not among patients with severe disease (managed with high-flow nasal oxygen, other non-invasive ventilation, or invasive mechanical ventilation/ extracorporeal membrane oxygenation). ${ }^{[25]}$

The subcommittee contextualised the results to the SA setting, given that our admission and treatment practices, and median hospital length of stay, differ from those in the study setting. Retrospective data from the DATCOV Sentinel Hospital Surveillance dataset (which includes all private hospitals and an increasing number of public sector hospitals) show a median hospital stay of $6-7$ days for COVID-19 patients in SA. ${ }^{[26]}$ However, currently patients requiring oxygen therapy may be kept in hospital longer while completing a 10-day course of corticosteroids. SA's hospital utilisation patterns may therefore differ from those outlined in the ACTT-1 study.

The subcommittee concluded that the benefits of remdesivir in SA would be modest at best, and that these potential benefits need to be balanced against considerations of applicability, feasibility, costs and equity. Remdesivir is not yet registered by the South African Health Products Regulatory Authority, but is accessible on a namedpatient basis (in terms of section 21 of the Medicines and Related
Substances Act 101 of 1965). Both the originator and generic products are expensive, and global supply is unreliable. For these reasons, the subcommittee did not recommend the use of remdesivir in the state sector, except in the context of clinical trials, which would generate much-needed local data and address the question of mortality impact.

At the time of publication, the review was in the process of being updated owing to recent publication of a further RCT. ${ }^{[27]}$

\section{Corticosteroids for the treatment of severe COVID-19}

Corticosteroids were investigated as a treatment for COVID-19 based on their anti-inflammatory effects. Using a similar process to that described for remdesivir, the subcommittee conducted a rapid review of the evidence for corticosteroids in severe COVID-19.

The review identified a well-conducted, adequately powered RCT that compared dexamethasone with usual care in hospitalised patients. Dexamethasone reduced mortality in patients who required oxygen or invasive ventilation. ${ }^{[28]}$ Adverse effects were not reported. The subcommittee considered the evidence of benefit to be clinically relevant, and of moderate quality. On this basis, it was agreed that the potential clinical benefit in SA would be substantial. In contrast to remdesivir, corticosteroids (including injectable dexamethasone) are inexpensive, are widely available, and have been shown to reduce mortality. Given these considerations, the subcommittee recommended that corticosteroids be used in all COVID-19 patients who require oxygen or mechanical ventilation.

The paucity of evidence for the safety and efficacy of corticosteroids in people living with HIV and in children was acknowledged.

\section{Conclusions}

The current health crisis has driven changes to the process of making prompt essential medicine policy decisions for COVID-19 clinical care questions. Rapid reviews are feasible and have been conducted successfully in the SA healthcare environment. They are a useful way of evaluating the best available information to urgently address specific clinical questions under pandemic conditions. In addition, using an EtD framework enables structured consideration of potential resource implications, practical issues and healthcare equity, ensuring 
that justifiable policy decisions are reached. It is also envisioned that trust in the associated recommendations will be enhanced through readily accessible results of a robust and transparent decision-making process.

\section{Declaration. None.}

\section{Acknowledgements. None.}

Author contributions. TDL conceptualised the manuscript. TDL and SMM were the primary authors, and TDL incorporated co-author feedback. TDL and SMM contributed to the final drafting of the article. All authors provided critical feedback and contributed to the final manuscript. AGP is the chair and GR is the vice-chair of the NEMLC COVID-19 subcommittee.

Funding. TK is partly supported by the Research, Evidence and Development Initiative (READ-It). READ-It (project number 300342104) is funded by UK aid from the UK government; however, the views expressed do not necessarily reflect the UK government's official policies. Conflicts of interest. JN and HR are co-principal investigators for the World Health Organization-sponsored SOLIDARITY trial conducted in SA. TK is co-lead of the South African GRADE Network. RW is employed by Liberty Health (Pty) Ltd, a private health insurer operating in SA and across the broader African continent.

1. Wang Y, Jiang W, He Q, et al. Early, low-dose and short-term application of corticosteroid treatmen in patients with severe COVID-19 pneumonia: Single-center experience from Wuhan, China. medRxi 2020 (epub 12 March 2020). https://doi.org/10.1101/2020.03.06.20032342

2. Mehra MR, Ruschitzka F, Patel AN. Retraction - Hydroxychloroquine or chloroquine with or without a macrolide for treatment of COVID-19: A multinational registry analysis. Lancet 2020;395(10240):1820. https://doi.org/10.1016/S0140-6736(20)31324-6

3. Mehra MR, Desai SS, Kuy S, Henry TD, Patel AN. Retraction: Cardiovascular disease, drug therapy, and mortality in Covid-19. N Engl J Med 2020;382(26):2582. https://doi.org/10.1056/NEJMc2021225

4. Zhong H, Wang Y, Zhang Z-L, et al. Efficacy and safety of current therapeutic options for COVID-19lessons to be learnt from SARS and MERS epidemic: A systematic review and meta-analysis. Pharmacol lessons to be learnt from SARS and MERS epidemic: A systematic
Res 2020;157:104872. https://doi.org/10.1016/j.phrs.2020.104872

5. Herrera-Perez D, Haslam A, Crain T, et al. A comprehensive review of randomized clinical trials in three 5. Herrera-Perez D, Haslam A, Crain T, et al. A comprehensive review of randomized clinical trials in three
medical journals reveals 396 medical reversals. eLife 2019;8:e45183. https://doi.org/10.7554/eLife. 45183 medical journals reveals 396 medical reversals. eLife 2019;8:e45183. https://doi.org/10.7554/eLife. 45183
6. National Department of Health, South Africa. National Drug Policy for South Africa. Pretoria: NDoH, 1996. https://www.sapc.za.org/Media/Default/Documents/Reference\%20-\%20National\%20Drug\%20 Policy\%20for\%20South\%20Africa.pdf (accessed 20 August 2020).

7. Perumal-Pillay VA, Suleman F. Understanding the decision making process of selection of medicines in the private sector in South Africa - lessons for low-middle income countries. J Pharm Policy Pract 2020;13:17. https://doi.org/10.1186/s40545-020-00223-5

8. South Africa. Medical Schemes Act 131 of 1998 - Explanatory Notes and Definitions to Annexure A. https://www.medicalschemes.com/files/Acts\%20and\%20Regulations/MSREGS19July2004.pdf (accessed 20 August 2020).

9. National Department of Health/National Institute of Communicable Diseases, South Africa. Clinical management of suspected or confirmed COVID-19 disease, version 5. 24 August 2020. http:// www.health.gov.za/index.php/component/phocadownload/category/628-clinical-management-ofsuspected-or-confirmed-covid-19-disease (accessed 25 August 2020).

10. Lavis JN, Permanand G, Oxman AD, Lewin S, Fretheim A. SUPPORT Tools for evidence-informed health Policymaking (STP) 13: Preparing and using policy briefs to support evidence-informed policymaking. Health Res Policy Syst 2009;7(1):S13. https://doi.org/10.1186/1478-4505-7-S1-S13

11. World Health Organization. WHO Handbook for Guideline Development. 2nd ed. Geneva: WHO, 2014. https://apps.who.int/iris/handle/10665/145714 (accessed 25 August 2020).

12. World Health Organization. Rapid Reviews to Strengthen Health Policy and Systems: A Practical Guide. Geneva: WHO, 2017. https://www.who.int/alliance-hpsr/resources/publications/rapidreview-guide/en/ (accessed 20 August 2020).

13. Garritty C, Gartlehner G, Kamel C, et al. Cochrane Rapid Reviews. Interim Guidance from the Cochrane Rapid Reviews Methods Group. March 2020. https://methods.cochrane.org/rapidreviews/ sites/methods.cochrane.org.rapidreviews/files/public/uploads/cochrane_rr_-_guidance23mar2020-final.pdf (accessed 20 August 2020).

14. National Department of Health, South Africa. National Essential Medicines List Committee protocol template for rapid reviews for COVID-19. Pretoria: NDoH, 2020. http://www.health. gov.za/index.php/national-essential-medicine-list-committee-nemlc/category/677-governance gov.za/index.php/national
(accessed 20 August 2020).

15. Cochrane South Africa. South African GRADE Network. 2020. https://southafrica.cochrane.org/ our-work/south-african-grade-network (accessed 25 August 2020).

16. Guyatt GH, Oxman AD, Schünemann HJ, Tugwell P, Knottnerus A. GRADE guidelines: A new series of articles in the Journal of Clinical Epidemiology. J Clin Epidemiol 2011;64(4):380-382. https://doi.org/10.1016/j.jclinepi.2010.09.011

17. Andrews J, Guyatt G, Oxman AD, et al. GRADE guidelines: 14. Going from evidence to recommendations: The significance and presentation of recommendations. J Clin Epidemiol 2013;66(7):719-725. https://doi.org/10.1016/j.jclinepi.2012.03.013

18. Alonso-Coello P, Oxman AD, Moberg J, et al. GRADE Evidence to Decision (EtD) frameworks: A systematic and transparent approach to making well informed healthcare choices. 2: Clinical practice guidelines. BMJ 2016;353:i2089. https://doi.org/10.1136/bmj.i2089

19. Alonso-Coello P, Schünemann HJ, Moberg J, et al. GRADE Evidence to Decision (EtD) frameworks: A systematic and transparent approach to making well informed healthcare choices. 1: Introduction. BMJ 2016;353:i2016. https://doi.org/10.1136/bmj.i2016

20. National Department of Health, South Africa. COVID-19 rapid reviews. 2020. http://www.health. gov.za/index.php/national-essential-medicine-list-committee-nemlc/category/633-covid-19-rapidgov.za/index.php/national-essential-
reviews (accessed 20 August 2020).

21. Sheahan TP, Sims AC, Leist SR, et al. Comparative therapeutic efficacy of remdesivir and combination lopinavir, ritonavir, and interferon beta against MERS-CoV. Nat Commun 2020;11(1):222. https:// doi.org/10.1038/s41467-019-13940-6

22. Wang $\mathrm{M}$, Cao R, Zhang $\mathrm{L}$, et al. Remdesivir and chloroquine effectively inhibit the recently emerged novel coronavirus (2019-nCoV) in vitro. Cell Res 2020;30(3):269-271. https://doi.org/10.1038/ s41422-020-0282-0

23. De Wit E, Feldmann F, Cronin J, et al. Prophylactic and therapeutic remdesivir (GS-5734) treatment in the rhesus macaque model of MERS-CoV infection. Proc Natl Acad Sci USA 2020;117(12):67716776. https://doi.org/10.1073/pnas.1922083117

24. Wang Y, Zhang D, Du G, et al. Remdesivir in adults with severe COVID-19: A randomised, double-blind, placebo-controlled, multicentre trial. Lancet 2020;395(10236):1569-1578. https://doi. org/10.1016/S0140-6736(20)31022-9

25. Beigel JH, Tomashek KM, Dodd LE, et al. Remdesivir for the treatment of Covid-19 - preliminary report. N Engl J Med 2020 (epub 22 May 2020). https://doi.org/10.1056/NEJMoa2007764

26. National Institute for Communicable Diseases. COVID-19 sentinel hospital surveillance update, . National Institute for Communicable Diseases. COVID-19 sentinel hospital surveillance update,
week 28. Johannesburg: NICD, 2020. https://www.nicd.ac.za/wp-content/uploads/2020/07/ week 28. Johannesburg: NICD, 2020. https://www.nicd.ac.za/wp-content/uploads/2020/07/
NICD-COVID-19-Weekly-Sentinel-Hospital-Surveillnace-update-Week-28-2020-final.pdf (accessed 20 August 2020)

27. Spinner CD, Gottlieb RL, Criner GJ, et al. Effect of remdesivir vs standard care on clinical status at 11 days in patients with moderate COVID-19: A randomized clinical trial. JAMA 2020 (epub 21 August 2020). https://doi.org/10.1001/jama.2020.16349

28. Horby P, Lim WS, Emberson JR, et al. Dexamethasone in hospitalized patients with Covid-19 preliminary report. N Engl J Med 2020 (epub 17 July 2020). https://doi.org/10.1056/NEJMoa2021436

Accepted 8 September 2020. 